\title{
Severe intellectual disability-progressive spastic diplegia syndrome
}

INSERM

\section{Source}

INSERM. (1999). Orphanet: an online rare disease and orphan drug data base. Severe intellectual disability-progressive spastic diplegia syndrome. ORPHA:404473

Severe intellectual disability-progressive spastic diplegia syndrome is a rare, genetic, syndromic intellectual disability disorder characterized by intellectual disability, significant motor delay, severe speech impairment, early-onset truncal hypotonia with progressive distal hypertonia/spasticity, microcephaly, and behavioral anomalies (autistic features, aggression or auto-aggressive behavior, sleep disturbances). Variable facial dysmorphism includes broad nasal tip with small alae nasi, long and/or flat philtrum, thin upper lip vermillion. Visual impairment (strabismus, hyperopia, myopia) is commonly associated. 